\title{
Safety and efficacy of azacitidine in Belgian patients with high-risk myelodysplastic syndromes, acute myeloid leukaemia, or chronic myelomonocytic leukaemia: results of a real-life, non-interventional post-marketing survey
}

\author{
Y. Beguin'1, D. Selleslag ${ }^{2}$, S. Meers ${ }^{3}$, C. Graux ${ }^{4}$, G. Bries ${ }^{5}$, D. Deeren ${ }^{6}$, \\ I. Vrelust ${ }^{7}$, C. Ravoet $^{8}$, K. Theunissen 9 , V. Voelter $^{10}$, H. Potier ${ }^{\dagger 10}$, \\ F. Trullemans ${ }^{11}$, L. Noens ${ }^{12}$, P. Mineur ${ }^{13}$
}

${ }^{1}$ Avenue de l'Hopital 1, Liège, Belgium, ${ }^{2}$ AZ Sint-Jan, Brugge-Oostende, Belgium, ${ }^{3}$ AZ KLINA, Brasschaat, Belgium, ${ }^{4} \mathrm{CHU}$ Dinant-Godinne UCL Namur, Godinne (Yvoir), Belgium, ${ }^{5} \mathrm{AZ}$ Turnhout, Belgium, ${ }^{6} \mathrm{Hematology-}$ Medical Oncology, H.-Hartziekenhuis Roeselare-Menen vzw, Belgium, ${ }^{7}$ UZA, Edegem, Belgium, ${ }^{8}$ Centres hospitaliers Jolimont, Haine-Saint-Paul, Belgium, ${ }^{9}$ Virga Jesse, Hasselt, Belgium, ${ }^{10} \mathrm{C} e l g e n e$ International, Boudry, Switzerland, ${ }^{11}$ Departement Oncologie/Hematologie, UZ Brussel, Belgium, ${ }^{12}$ Ghent University Hospital, Belgium, ${ }^{13}$ Grand Hopital de Charleroi (GHdC), Belgium

Objectives: We evaluated azacitidine (Vidaza ${ }^{\oplus}$ ) safety and efficacy in patients with myelodysplastic syndrome (MDS), acute myeloid leukaemia (AML), and chronic myelomonocytic leukaemia (CMML), in a real-life setting. Treatment response, dose, and schedule were assessed.

Methods: This non-interventional, post-marketing survey included 49/50 patients receiving azacitidine at 14 Belgian haematology centres from 2010-2012. Treatment-emergent adverse events (TEAEs), including treatment-related TEAEs, and serious TEAEs (TESAEs) were recorded throughout the study. Treatment response [complete response (CR), partial response (PR), haematological improvement $(\mathrm{HI})$, stable disease (SD), treatment failure (TF)) and transfusion-independence (TI) were evaluated at completion of a 1-year observation period (1YOP) or at treatment discontinuation, and overall survival (OS), at study conclusion.

Results: The median age of patients was 74.7 (range: 43.9-87.8) years; $69.4 \%$ had MDS, $26.5 \%$ had primary or secondary AML, and $4.1 \%$ had CMML. Treatment-related TEAEs, grade 3-4 TEAEs, and TESAEs were reported in $67.3 \%, 28.6 \%$, and $18.4 \%$ of patients, respectively. During 1YOP, patients received a median of 7 (1-12) treatment cycles. Treatment response was assessed for 38/49 patients. Among MDS and CMML patients $(n=29), 41.4 \%$ had $\mathrm{CR}$, PR, or $\mathrm{HI}, 41.4 \%$ had SD, and $17.2 \%$ had TF. Among AML patients $(n=9), 44.4 \%$ had CR or PR, $33.3 \%$ had SD, and $22.2 \%$ had TF. TI was observed in $14 / 32(43.8 \%)$ patients who were transfusion-dependent at baseline. Median (95\% confidence interval) OS was 490 (326-555) days; 1-year OS estimate was $0.571(0.422-0.696)$.

Conclusions: Our data support previous findings that azacitidine has a clinically acceptable safety profile and shows efficacy.

Keywords: Azacitidine, Myelodysplastic syndromes, Acute myeloid leukaemia, Chronic myelomonocytic leukaemia

\section{Introduction}

Myelodysplastic syndromes (MDSs) are haematopoietic stem cell disorders characterized by ineffective

Correspondence to: Y. Beguin, Avenue de l'Hopital 1, GIGA, B34, B-4000 Liège, Belgium. Email: yves.beguin@chu.ulg.ac.be

${ }^{\dagger}$ Author's affiliation at the time of manuscript preparation. haematopoiesis and peripheral blood cytopenias. ${ }^{1-4}$ The median age at MDS diagnosis is $\geq 70$ years. ${ }^{5-7}$ MDS prognosis depends on disease risk features assessed by the International Prognostic Scoring System (IPSS): patients with low or intermediate-1 (Int-1) IPSS scores have a median overall survival (OS) of several years, whereas patients with intermediate-2 
(Int-2) or high IPSS scores have a median OS of approximately 4 months. $^{8}$ In total, $30-50 \%$ of MDS cases progress to acute myeloid leukaemia (AML) ${ }^{9-11}$ Chronic myelomonocytic leukaemia (CMML), initially classified morphologically as a MDS by the French-American-British cooperative group, ${ }^{12}$ was later classified by the World Health Organization (WHO) (2008) as a mixed MDS/ myeloproliferative neoplasm; ${ }^{4}$ MDS with $20-30 \%$ bone marrow (BM) blast cells was classified as AML by the WHO. ${ }^{4}$ Similarly to MDS, CMML and AML are also commonly diagnosed later in life. ${ }^{13}$

Currently, the only curative option for MDS is allogeneic haematopoietic stem cell transplantation (allo-HSCT). ${ }^{14-17}$ However, due to high treatmentrelated morbidity and mortality, this procedure is limited to young patients with good performance status. Thus, since MDS is a disease of the elderly, only a minority of MDS patients are transplant candidates. $^{8,18,19}$

In a phase III randomised trial (AZA-001), the OS of higher-risk MDS patients (Int-2 or highrisk; IPSS $=1.5-2$ or $\geq 2.5$, respectively) was significantly prolonged upon treatment with a pyrimidine nucleoside analogue, azacitidine (Vidaza ${ }^{\circledR}$; Celgene Corporation, Summit, NJ, USA), compared with conventional care (median OS: 24.5 vs. 15.0 months; $P=0.0001){ }^{20}$ These findings led to the approval of azacitidine by the European Medicines Agency (EMA) in December 2008 for treatment of Int-2 and high-risk MDS (according to IPSS), AML with 20-30\% BM blasts (according to WHO 2008), and CMML with 10-29\% BM blasts and no myeloproliferative syndrome (i.e. $<13000 / \mu 1$ white blood cells) for patients who are not transplant eligible and who have not received previous therapies. The indications for azacitidine treatment, as well as the reimbursement criteria, have been recently described in detail in Meers et $a l^{21}$

To date, post-marketing data assessing the safety and efficacy of azacitidine in real-life settings have been limited. Here, we report the results of a real-life 'Belgian non-interventional post-marketing survey in MDS, AML or CMML patients treated with Azacitidine evaluating Safety and Efficacy' (BASE).

\section{Patients and Methods}

\section{Study design and setting}

In this non-interventional post-marketing survey, patients were recruited in 14 Belgian haematology centres from 2010 to 2012. The decision on azacitidine treatment was made prior to the study enrolment. Treatment was prescribed and continued at discretion of the treating physician according to Belgian clinical practice. Patients were observed for approximately 1 year following treatment initiation. Patients treated for less than 1 year were observed up to 28 days after the last azacitidine administration. At study end, survival information was collected for all patients. Written informed consent was obtained from all patients before recruitment.

The protocol and associated documents were reviewed and approved by the National Competent Authority and National/Local Ethics Committees in accordance with local regulations. This study was conducted in accordance with Good Clinical Practice and all applicable regulatory requirements, including the Declaration of Helsinki.

\section{Patients and data collection}

Adult patients with higher-risk MDS, primary or secondary AML (based on assessed medical histories and on laboratory data, including BM biopsy and/or BM aspirate, peripheral blood count, peripheral blood smear and BM cytogenetics), or CMML, treated with azacitidine according to Belgian reimbursement criteria and who provided written informed consent, were eligible for the study. Patients were excluded if they were participating in an interventional clinical trial at the time of recruitment.

Patient and disease characteristics were collected at baseline using case record forms (CRFs), completed by the physician. The following baseline patient characteristics were collected: age, gender, and Eastern Cooperative Oncology Group performance status. ${ }^{22}$ The following baseline disease characteristics were collected: disease diagnosis according to the WHO 2008 classification, ${ }^{4}$ time since diagnosis, percentage of BM blasts, cytopenias, packed red blood cell (PRBC) transfusion within 8 weeks before treatment initiation, cytogenetic abnormalities, and IPSS score (assessed for MDS patients). ${ }^{23}$

At each treatment cycle, dosing and dose modification details, adverse events (AEs), supportive treatments, and haematological parameters were recorded by the investigator in a CRF.

\section{Study objectives}

The primary objective of this real-life study was to assess the safety of azacitidine in Belgian MDS, AML, or CMML patients. The secondary objectives included assessment of the efficacy of azacitidine in terms of treatment response and OS, and evaluation of the treatment dose and schedule.

\section{Treatment and administration}

Patients were treated with azacitidine according to the schedule used in Belgian clinical practice: $75 \mathrm{mg} /$ $\mathrm{m}^{2} /$ day for 7 days every 28 days. The decision of treatment discontinuation was made by the patient or the investigator. The reason for treatment discontinuation was recorded in the CRF. 


\section{Safety assessment}

Treatment-emergent AEs (TEAEs) and serious TEAEs (TESAEs) were recorded up to 28 days following the last treatment dose administered. TEAEs were defined as AEs with onset at or after the first treatment administration. TESAEs were defined as AEs with onset at or after the first treatment administration that resulted in death, congenital anomaly or birth defect, persistent or significant disability/incapacity, were life-threatening or required hospitalisation or prolongation of hospitalisation.

The severity of all AEs was assessed by the investigators on a 1-5 scale, according to the Common Terminology Criteria for Adverse Events version $4.0 .^{24}$ Symptoms of grade 3-4 intensity were defined as AEs resulting in a marked (grade 3) or significant (grade 4) limitation in activity, and requiring medical intervention, therapy, or hospitalisation. Grade 5 symptoms were defined as AEs leading to death. The causality of all AEs was assessed by the investigators. Following an AE, the decision of treatment discontinuation or treatment dose reduction was made by the investigators.

TESAEs that had not resolved upon a patient's discontinuation of participation in the study were followed until recovery, recovery with sequelae, nonrecovery (death due to another cause), or death (due to TESAE).

\section{Treatment administration and exposure variables}

The following treatment administration variables were assessed during the 1-year observation period: mean dose per treatment day, number of treatment days/cycle, cycle duration, number of cycles, and number of cycles with dose interruption or reduction. Dose interruption was defined as treatment administration for $<7$ days, and dose reduction as administration of $<80 \%$ of the planned dose intensity for a single cycle.

\section{Efficacy assessment}

At the end of the 1-year observation period or at treatment discontinuation, azacitidine efficacy in terms of treatment response, transfusion independence (TI), and time-to-treatment discontinuation were assessed for all evaluable patients (38 patients for treatment response; 32 for TI; 49 for time-to-treatment discontinuation). Treatment response was evaluated, according to the International Working Group 2000 criteria for $\mathrm{MDS}^{25}$ and the revised 2003 criteria for $\mathrm{AML},{ }^{26}$ as complete response (CR), CR with incomplete blood count recovery (CRi) (AML patients), partial response (PR), haematological improvement (HI; MDS, and CMML patients), stable disease (SD), or treatment failure (TF). Overall treatment response was defined as achievement of CR, PR, or HI (MDS and CMML patients) or CR, CRi, or PR (AML patients). TI was defined as the absence of PRBC transfusion during 2 consecutive cycles for patients who had received such transfusion within 8 weeks before the azacitidine treatment initiation. Time-totreatment discontinuation was defined as number of days between the first treatment dose administration and treatment discontinuation. Survival was defined in all evaluable patients as the time between the first administration date and the last contact alive (performed about 1 year after the treatment initiation of the last patient enrolled) or date of death. Time-totreatment discontinuation and 1-year OS were calculated using Kaplan-Meier curves. An additional analysis evaluated the OS according to treatment response, i.e. in responders, patients with SD and nonresponders. Responders were defined as patients with $\mathrm{CR}, \mathrm{PR}, \mathrm{CRi}$, or $\mathrm{HI}$, and non-responders as patients with TF.

\section{Statistical analyses}

All statistical analyses were performed on the safety cohort, which included all patients who received at least one treatment dose. Continuous variables were determined as mean ( \pm standard deviation), median (range: minimum-maximum), and number of observations. Kaplan-Meier analysis was used to describe time-to-event data. All statistical analyses were performed using the SAS package for Windows, version 9.2 .

\section{Results}

\section{Baseline patient and disease characteristics}

Fifty patients treated with azacitidine in routine clinical practice were recruited. One patient initially included in the intent-to-treat population was lost to follow-up; the final safety cohort comprised 49 patients (Fig. 1). The majority of patients $(77.5 \%)$ were $\geq 65$ years old, with a median age of 74.7 (43.987.8 ) years; $69.4 \%$ were male. The Eastern Cooperative Oncology Group (ECOG) performance status was 0 for $24.5 \%, 1$ for $44.9 \%$, and 2 for $18.4 \%$ of patients (Supplementary Material $1 \mathrm{http} / / / \mathrm{dx}$.doi.org/ 10.1179/2295333714Y.0000000102.s1). The WHO diagnosis was MDS for $34(69.4 \%)$, primary or secondary AML for $13(26.5 \%)$ and CMML for two $(4.1 \%)$ patients. $65.3 \%$ of patients had received PRBC transfusion within 8 weeks before the treatment initiation. The mean time since diagnosis was 10 $( \pm 14.68)$ months (Supplementary Material $1 \mathrm{http}: / /$ dx.doi.org/10.1179/2295333714Y.0000000102.s1).

Among the 34 MDS patients, $2.9 \%$ had refractory cytopenia with multilineage dysplasia, $32.4 \%$ had refractory anaemia with excess of blasts-1 (RAEB-1), and $61.8 \%$ had RAEB-2. The majority of MDS patients had $>10 \%$ BM blasts $(55.9 \%)$ and poor karyotype score $(44.1 \%)$, resulting in an Int-2 IPSS 


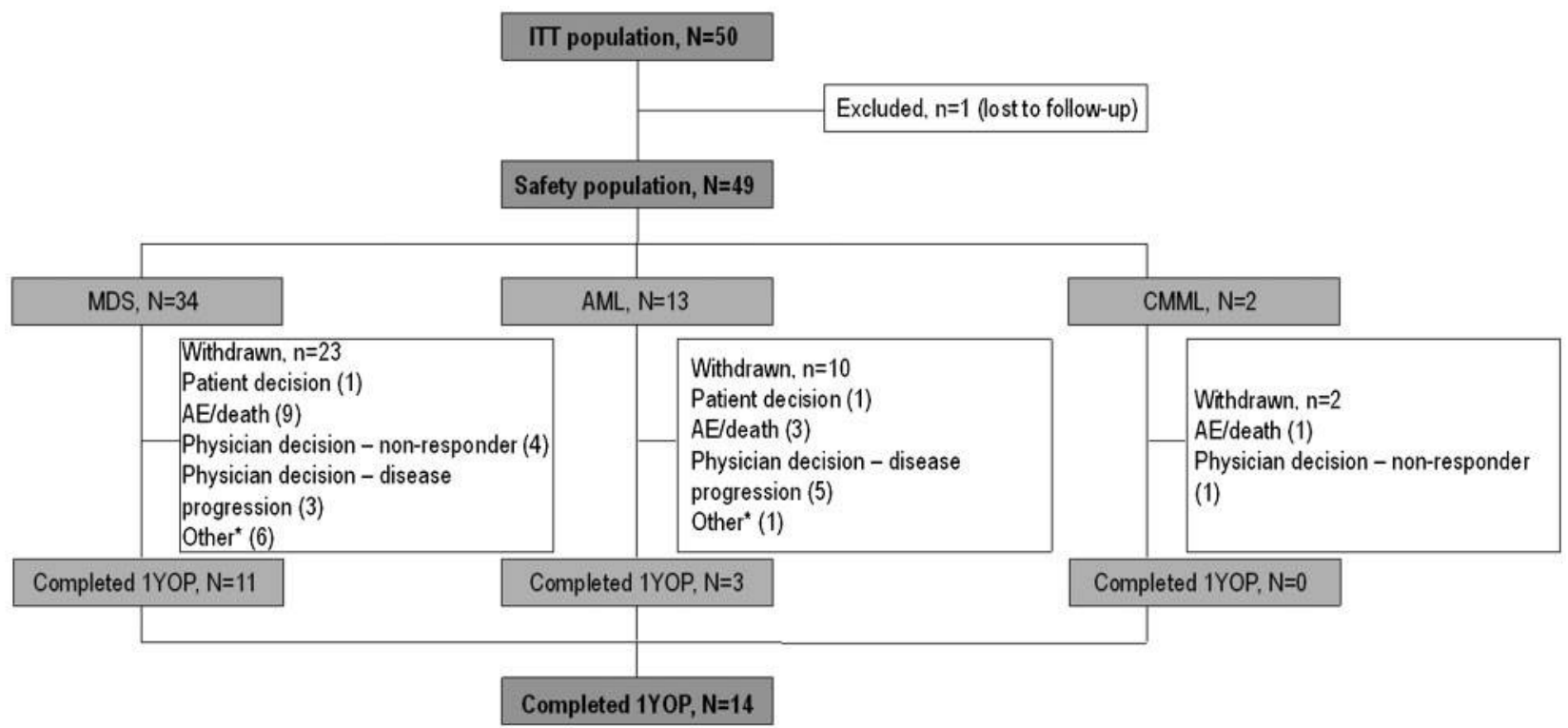

Figure 1 Participant flow. ITT, intent-to-treat; N, number of patients; $n$, number of patients within the group; MDS, myelodysplastic syndromes; AML, acute myeloid leukaemia; CMML, chronic myelomonocytic leukaemia; AE, adverse event; 1YOP, 1-year observation period. Other: allogenic stem cell transplantation (4); less than $5 \%$ blasts present (1); physician decision of not giving more than 6 cycles (1); no reimbursement of azacitidine (1).

score $(70.6 \%)$ (Supplementary Material 2 http:// dx.doi.org/10.1179/2295333714Y.0000000102.s2). 26.5\% had a normal karyotype and $26.5 \%$ had a complex karyotype (Supplementary Table S1 http://dx.doi.org/ 10.1179/2295333714Y.0000000102.s1).

Among the 13 AML (20-30\% BM blasts) patients, 7.7\% had AML with recurrent cytogenetic abnormalities, 53.8\% had AML with multilineage dysplasia with prior MDS, and 23.1\% had AML with multilineage dysplasia without prior MDS. Two of 9 evaluated AML patients $(22.2 \%)$ had a normal karyotype and $4(44.4 \%)$ had a complex karyotype.

\section{Safety}

TEAEs were reported in all patients (Table 1). TEAEs considered by the investigators as causally related to treatment were reported in $67.3 \%$ of patients (Table 1); the most common were general disorders and administration site reactions, gastrointestinal disorders, and haematological complications, reported in $40.8 \%, 38.8 \%$, and $30.6 \%$ of patients, respectively.
The most common general disorders and administration site reactions were fatigue and injection site reactions, reported in $16.3 \%$ and $14.3 \%$ of patients, respectively. The most common gastrointestinal disorders were nausea, diarrhoea, and vomiting, reported in $22.4 \%, 14.3 \%$, and $12.2 \%$ of patients, respectively. The most common haematological complications were febrile neutropenia and anaemia, each reported in $16.3 \%$ of patients. Treatment-related grade 3-4 TEAEs were reported in $28.6 \%$ of patients (Table 1 ). The most common were haematological complications, gastrointestinal disorders and infections, reported in $18.4 \%, 8.2 \%$, and $8.2 \%$ of patients, respectively. The most common haematological complication was febrile neutropenia ( $14.3 \%$ of patients), and the most common gastrointestinal disorder was diarrhoea ( $4.1 \%$ of patients). Treatment-related TESAEs were reported in $18.4 \%$ of patients (Table 1); the most common were haematological complications and infections, each reported in $12.2 \%$ of patients. TEAEs leading to death or discontinuation,

Table 1 Numbers and percentages of patients with treatment-emergent adverse events or serious adverse events

\begin{tabular}{|c|c|c|c|c|}
\hline & All $(N=49), n(\%)$ & MDS $(N=34), n(\%)$ & AML $(N=13), n(\%)$ & CMML $(N=2), n(\%)$ \\
\hline TEAEs & $49(100.0)$ & $34(100.0)$ & $13(100.0)$ & $2(100.0)$ \\
\hline Related* & $33(67.3)$ & $21(61.8)$ & $10(76.9)$ & $2(100.0)$ \\
\hline Related grade 3-4 & $14(28.6)$ & $10(29.4)$ & $3(23.1)$ & $1(50.0)$ \\
\hline TEAEs resulting in TD or death ${ }^{\dagger}$ & $11(22.4)$ & $8(23.5)$ & $2(15.4)$ & $1(50.0)$ \\
\hline TESAE & $22(44.9)$ & $15(44.1)$ & $6(46.2)$ & $1(50.0)$ \\
\hline Related* & $9(18.4)$ & $6(17.6)$ & $2(15.4)$ & $1(50.0)$ \\
\hline
\end{tabular}

Note: MDS, myelodysplastic syndromes; AML, acute myeloid leukaemia; CMML, chronic myelomonocytic leukaemia; $N$, number of patients; $n(\%)$, number (percentage) of patients within the category; TEAEs, treatment-emergent adverse events; TD, treatment discontinuation; TESAEs, treatment-emergent serious adverse events. *Considered by the investigators to be causally related to treatment.

†'Independent of disease progression. 
independently of disease progression, were reported in $22.4 \%$ of patients (Table 1 ).

\section{Treatment administration and exposure}

Within the 1-year observation period, patients received a median of 7 (1-12) treatment cycles, with a median cycle duration of 30.5 days and median treatment duration of 273 days; $28.6 \%$ of patients received 12 treatment cycles (Supplementary Material $3 \mathrm{http}: / /$ dx.doi.org/10.1179/2295333714Y.0000000102.s3). The median follow-up time was 272 days; $71.4 \%$ of patients did not complete the 1 -year observation period. The main reasons for treatment discontinuation were $\mathrm{AE} /$ death $(37.1 \%)$, disease progression $(22.9 \%)$, and nonresponse $(14.3 \%)$. Other reasons were allogenic stem cell transplantation, less than $5 \%$ blasts present in the BM, physician decision of not giving more than 6 cycles, patient decision, and no reimbursement of azacitidine because of an insufficient IPSS score. For the 13 patients who discontinued the treatment due to AE/death, 6 discontinued the treatment due to death, and 7 due to AEs; $24.5 \%$ of patients had $\geq 1$ cycle with dose reduction and $24.5 \% \geq 1$ cycle with dose interruption.

\section{Efficacy}

\section{Treatment response, time-to-treatment} discontinuation and transfusion independence

Treatment response was evaluated for 38/49 patients. For 11 patients, the best response was not assessed by the investigators; of those, within the observation period, 1 patient received 1 cycle, 2-2 cycles, 3-3 cycles, $2-4$ cycles, $1-5$ cycles, and $2-12$ cycles. Two of these patients completed the 1-year observation period, and 9 discontinued the study due to: patient decision (1), AE/death (6), or disease progression (2). Overall treatment response was achieved in 12/29 (41.4\%) MDS and CMML patients and in 4/9 (44.4\%) AML patients (Table 2). Overall, 14/49 (28.6\%) patients completed the 1-year observation period. The median time-to-treatment discontinuation due to non-response, disease progression, AE, or death was 308 days for all patients (Fig. 2A), 304 days for MDS patients, and 329 days for AML patients (Fig. 2B).
Among the 32 patients who were transfusion dependent before study start, $43.8 \%$ became TI; 1 was TI during 2 cycles, 4 during 3 cycles, 3 during 4 cycles, 3 during 5 cycles, 1 during 6 cycles, 1 during 7 cycles, and 1 during 10 cycles.

\section{Overall survival}

The median OS (based on date of last contact or date of death) since the first azacitidine administration was 490 days (95\% confidence interval: 326-555) for all patients (Fig. 3A), 501 days (317-665 days) for MDS patients and 363 days (115-576 days) for AML patients (Fig. 3B). The estimate of the 1-year OS probability was $0.57(0.42-0.70)$ for all patients, 0.59 (0.41-0.73) for MDS patients, and $0.46(0.19-0.70)$ for AML patients.

In the additional analysis of the OS according to the response to treatment, the median OS estimates were 576 days for responders $(n=16), 513$ days for patients with SD $(n=15)$, and 269 days for nonresponders $(n=7)$ (Fig. $3 \mathrm{C})$.

\section{Discussion}

Two previously published, phase III randomised clinical trials showed significantly longer OS and lower rate of progression to AML in higher-risk (43\% Int-2; 46\% high-risk) ${ }^{20}$ or Int-1 to high-risk (45\% Int-1; 27\% Int-2; $19 \%$ high-risk $)^{27}$ MDS patients treated with azacitidine compared to conventional treatments. However, in routine clinical practice, physicians often deal with elderly patients with co-morbidities and a poor performance status, who are often ineligible for randomised clinical trials. Therefore, non-interventional surveillance studies are needed to collect and describe real-life data. Previous real-life studies assessed azacitidine efficacy in French [patient-named programme: authorization for temporary utilization (ATU)], Dutch, Turkish, Italian, Scottish or Austrian patients with MDS, AML, or CMML. ${ }^{28-34}$ The Scottish, Italian and Turkish studies also evaluated the azacitidine safety profile. The current survey evaluated safety and efficacy of azacitidine in Belgian MDS, AML, or CMML patients treated in a real-life setting.

Table 2 Treatment response

\begin{tabular}{lclcl}
\hline & MDS $(\boldsymbol{N}=\mathbf{2 7}), \boldsymbol{n}(\mathbf{\%})$ & CMML $(\boldsymbol{N}=\mathbf{2}), \boldsymbol{n}(\%)$ & MDS + CMML $(\boldsymbol{N}=\mathbf{2 9}), \boldsymbol{n}(\%)$ & AML $(\boldsymbol{N}=\mathbf{9}), \boldsymbol{n}(\%)$ \\
\hline Overall response* & $12(44.4)$ & $0(0.0)$ & $12(41.4)$ & $4(44.4)$ \\
CR/CRi & $3(11.1)$ & $0(0.0)$ & $3(10.3)$ & $4(44.4)$ \\
PR & $3(11.1)$ & $0(0.0)$ & $3(10.3)$ & $0(0.0)$ \\
HI & $6(22.2)$ & $0(0.0)$ & $6(20.7)$ & $\ldots$ \\
SD & $11(40.7)$ & $1(50.0)$ & $12(41.4)$ & $3(33.3)$ \\
TF & $4(14.8)$ & $1(50.0)$ & $5(17.2)$ & $2(22.2)$ \\
\hline
\end{tabular}

Note: MDS, myelodysplastic syndromes; CMML, chronic myelomonocytic leukaemia; AML, acute myeloid leukaemia; $N$, number of patients; $n(\%)$, number (percentage) of patients within the category; CR, complete response (MDS and CMML patients); CRi, complete response with incomplete blood count recovery (AML patients); PR, partial response; HI, haematological improvement; SD, stable disease; TF, treatment failure.

*For MDS + CMML patients: CR+PR+HI; for AML patients, CR+PR+CRi. 
A.

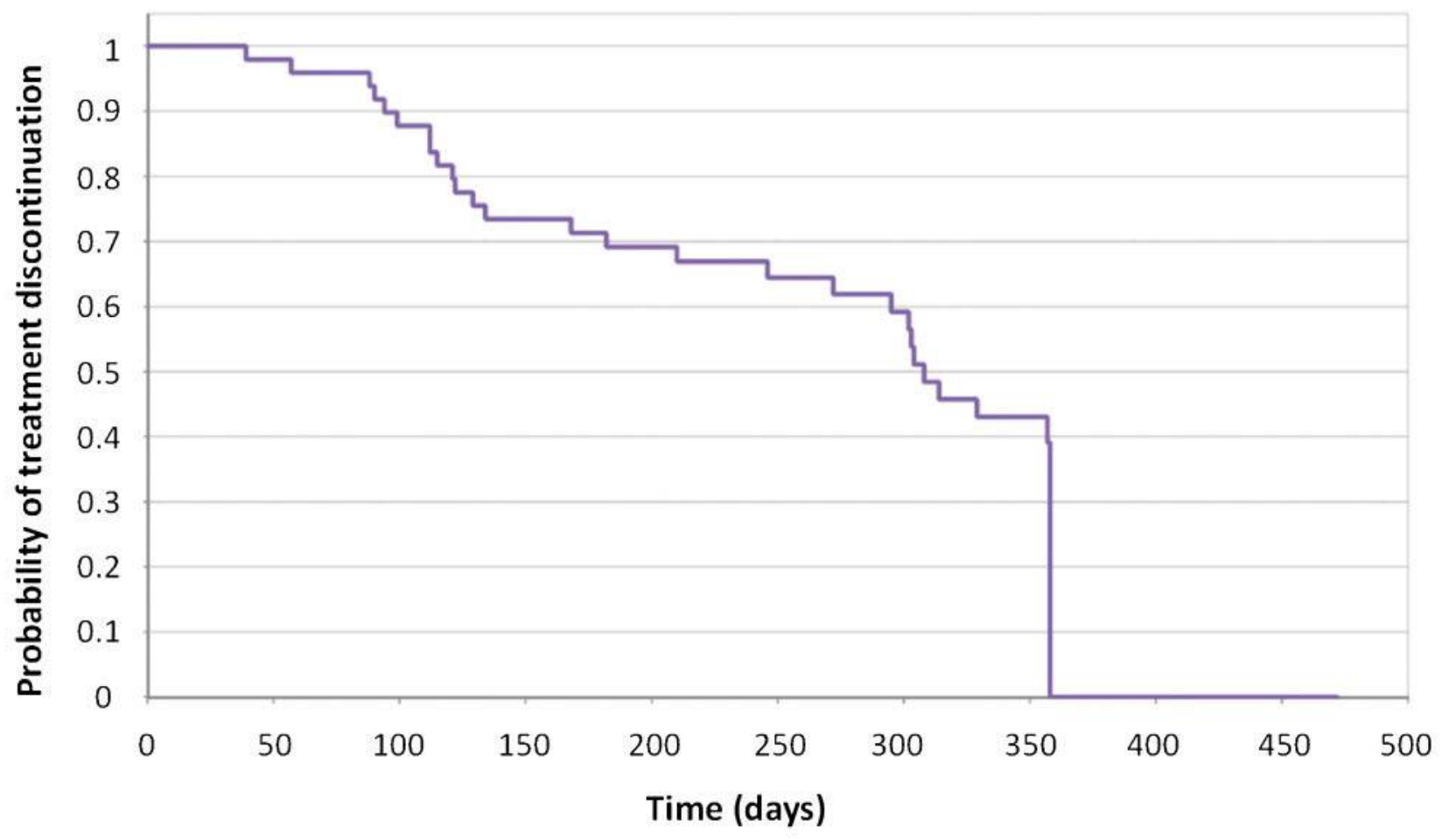

B.

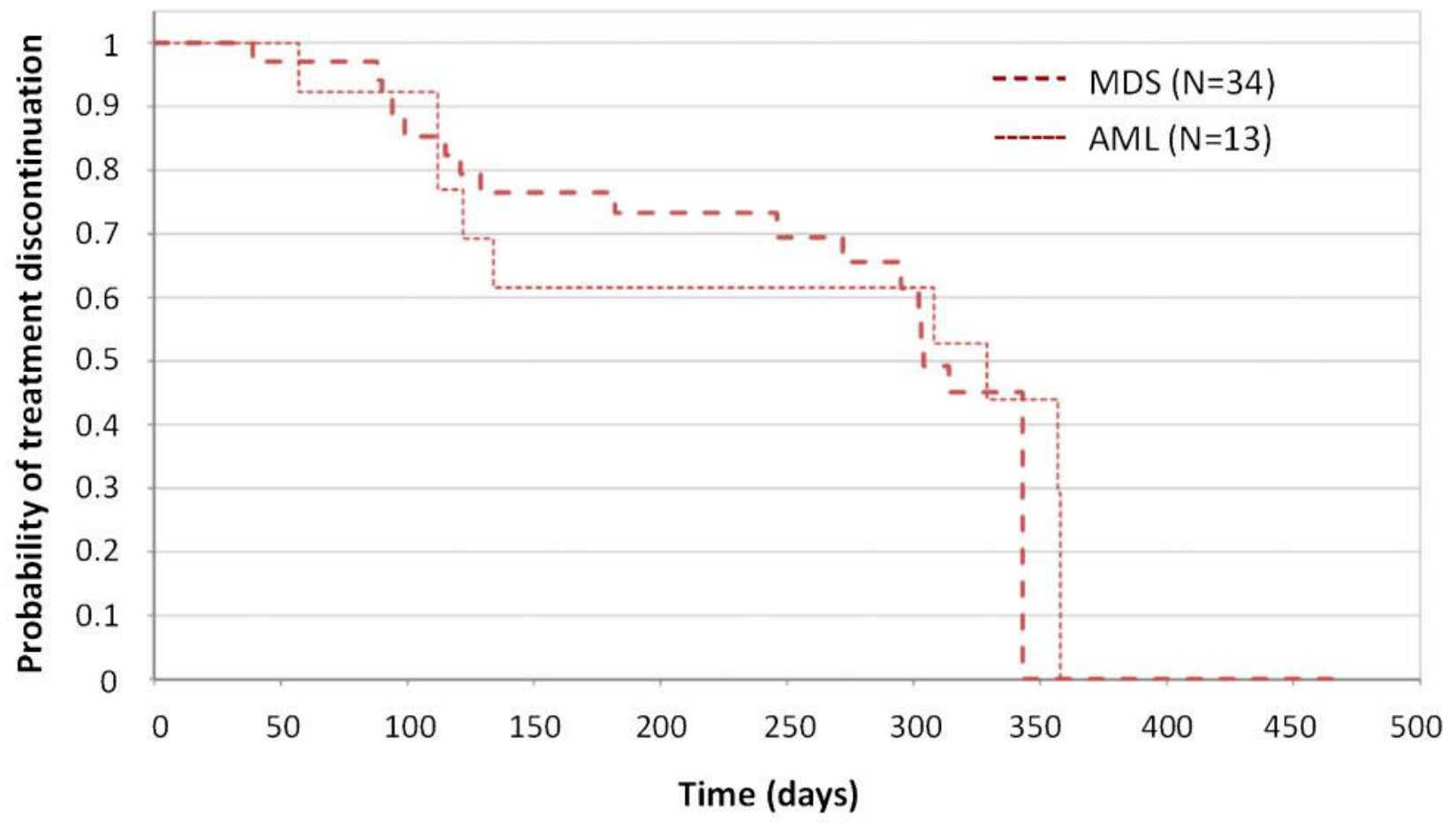

Figure 2 Time-to-treatment discontinuation (A) in all patients and (B) in MDS and AML patients. MDS, myelodysplastic syndromes; AML, acute myeloid leukaemia; $N$, number of patients.

In this survey, patients were slightly older [median age: 74.7 (43.9-87.8) years] than patients included in the previous real-life studies with azacitidine [median age: $70-73$ (20-91 for all studies)] (Table 3). ${ }^{28,29,31-34}$
Among the MDS patients included in this survey, there were more Int-2 (70.6\%) and less high-risk patients $(20.6 \%)$ compared to the French ATU study (54\% Int-2 and $43 \%$ high-risk), ${ }^{29}$ probably due to 
A.

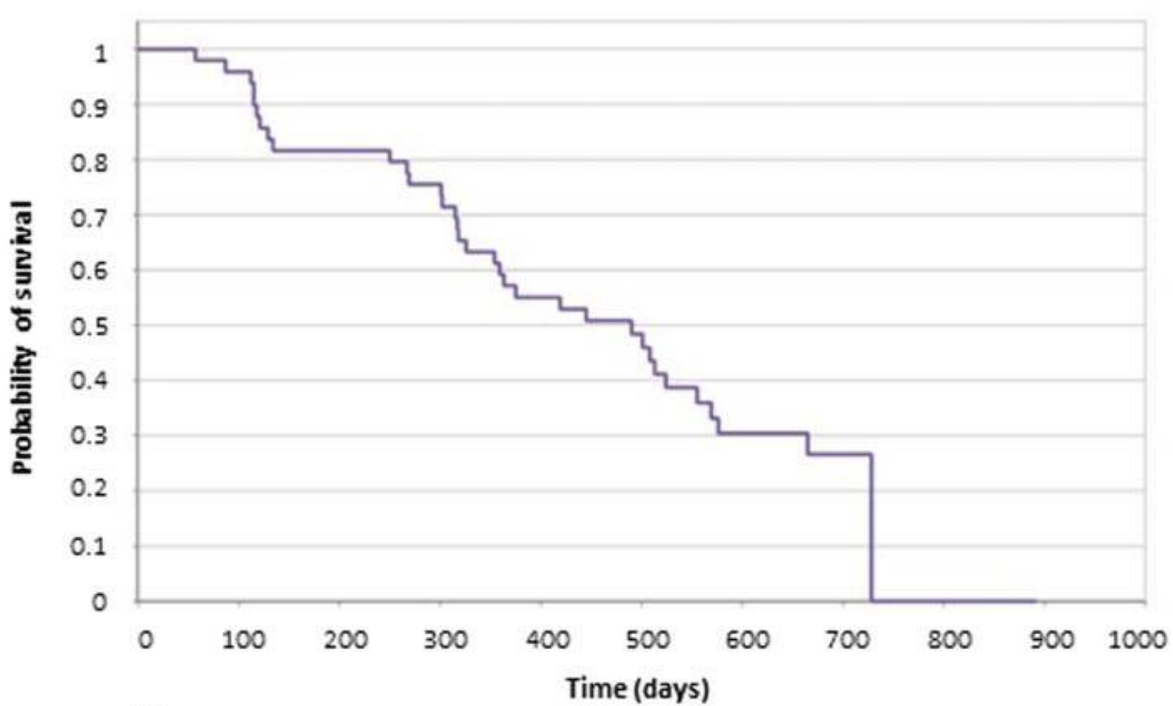

B.

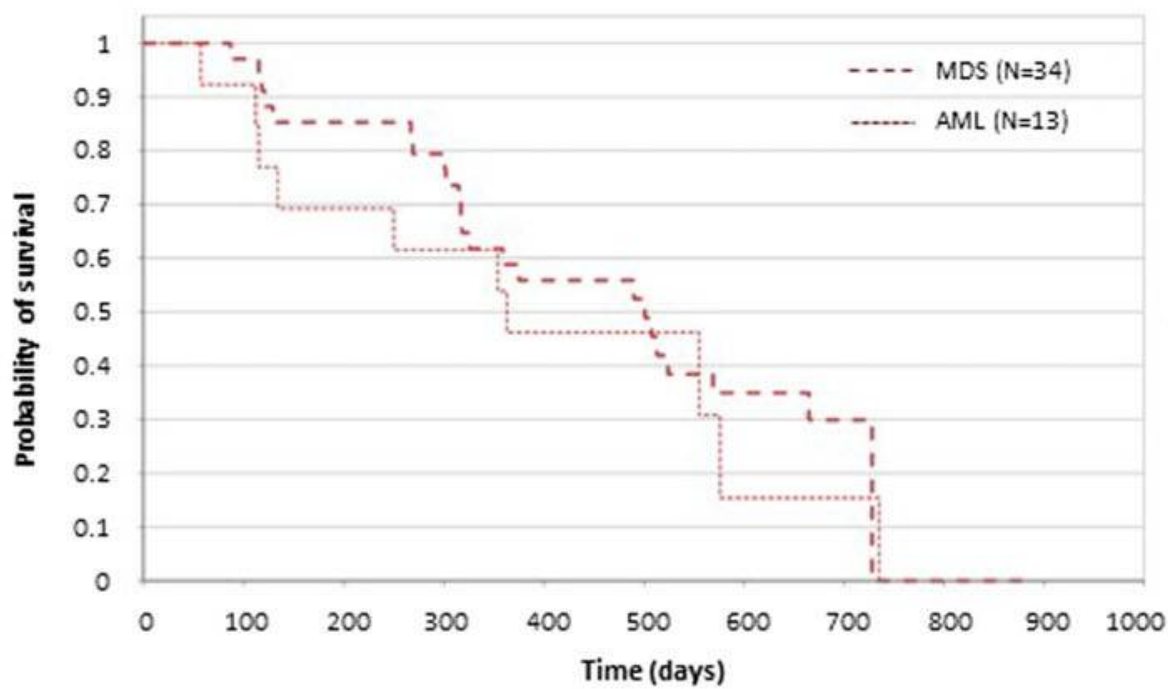

C.

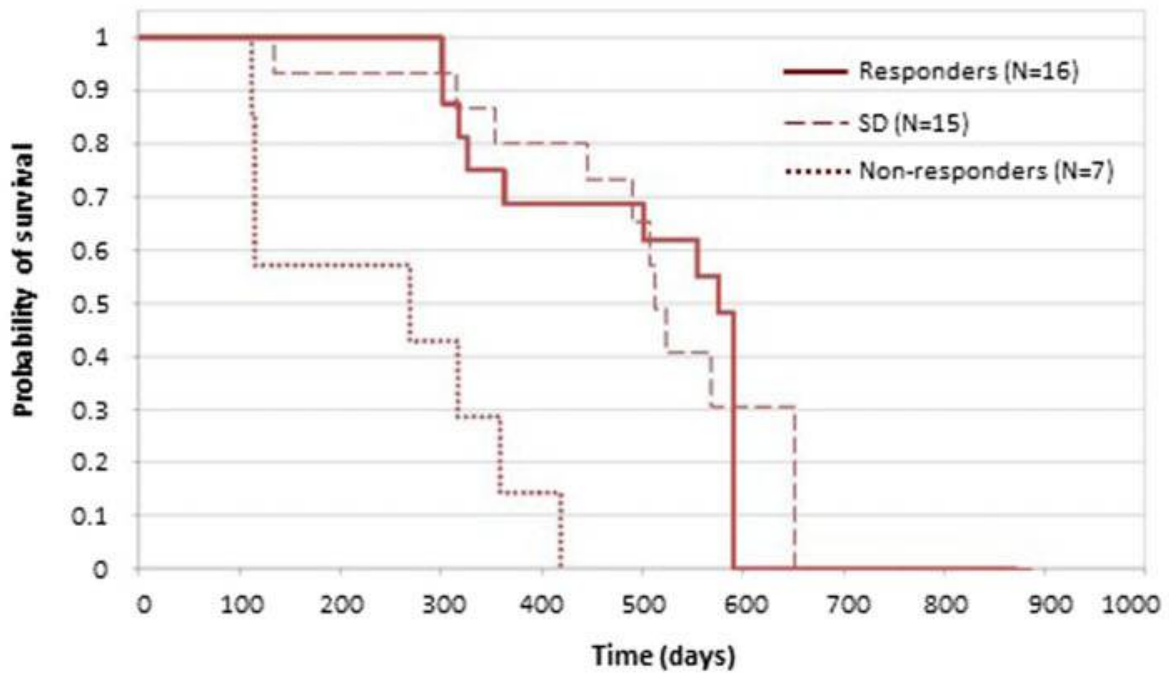

Figure 3 Overall survival (A) in all patients, (B) in MDS and AML patients and (C) in patients according to the response to treatment. MDS, myelodysplastic syndromes; AML, acute myeloid leukaemia; SD, stable disease; N, number of patients. Responders were defined as patients with complete remission, partial remission, complete remission with incomplete blood count recovery (acute myeloid leukaemia patients) or haematological improvement. Non-responders were defined as patients with treatment failure. 
different treatment strategies or referral patterns. The majority of MDS patients included in our and the previous real-life studies had poor karyotype score and RAEB-2 WHO diagnosis. ${ }^{29,34}$

The azacitidine safety profile was consistent with previous findings. ${ }^{20,28,31,33}$ The most common TEAEs were general disorders, administration site reactions, gastrointestinal disorders, and infections. Treatmentrelated and grade 3-4 TEAEs were also consistent with those reported in the AZA-001 trial. ${ }^{20}$

Belgian patients received azacitidine for a median of 7 (1-12) cycles within the 1-year observation period. The number of cycles was equivalent to the Italian study [median: 7 (2-22) cycles; follow-up not reported) and superior to the French ATU [median: 6 (1-52) cycles; median follow-up: 26 months], Dutch [median: 5 (1-19) cycles; median follow-up: 13 months], and the Austrian studies [median: 4 (1-24) cycles; follow-up not reported). ${ }^{28,29,33,34}$ However, the ATU and the Dutch studies included a higher number of high-risk patients, who are more likely to discontinue treatment earlier due to haematological complications or death than Int-2 MDS patients.

The majority of Belgian patients received the US Food and Drug Administration- and EMA-approved azacitidine schedule $\left(75 \mathrm{mg} / \mathrm{m}^{2} /\right.$ day for 7 days every 28 days), and about $25 \%$ of patients received shorter schedules, mostly due to AE/death, disease progression, and non-response. The ATU study reported a similar proportion of patients with reduced treatment schedule $(28 \%){ }^{29}$ In this previous study, a nonsignificant trend for reduced OS was observed in patients receiving reduced schedules. In the Dutch study, the number of patients with a treatment response increased with the number of cycles received. ${ }^{34}$ Similarly, in a secondary analysis of the AZA-001 trial, 91\% of high-risk MDS patients responded to treatment within 6 azacitidine cycles and the continuation of treatment further improved the response rates. ${ }^{35}$

In our survey, approximately $42 \%$ of patients (41.4\% of high-risk MDS or CMML patients and $44.4 \%$ of AML patients) achieved a treatment response, which is consistent with findings of the previous Dutch, French, Italian, and Austrian studies, in which the overall treatment responses were $48 \%, 43 \%, 50 \%$, and $45 \%$, respectively (Table 3 ). ${ }^{28,29,33,34}$ In our survey, the overall response was higher than that reported in the Scottish study in patients with MDS or AML $(31 \%){ }^{31}$ This could be due to the higher number of azacitidine cycles (median: 7 cycles) compared to the Scottish study (median: 4 cycles), as the response to azacitidine may appear only after several treatment cycles. In our survey, the median OS was 490 days (16.1 months). This was shorter than the median OS of 24.5 months reported in the AZA-001 trial, but comparable to the median OS of 13.5, 13, and 15 months observed in the French, Dutch, and Italian

Table 3 Patient characteristics and efficacy of azacitidine - comparison of results from the current and previously published studies

\begin{tabular}{|c|c|c|c|c|c|c|c|c|}
\hline Study & $\begin{array}{l}\text { BASE } \\
\text { Current study }\end{array}$ & $\begin{array}{l}\text { AZA-001 } \\
(A Z A \text { arm })^{20}\end{array}$ & $\begin{array}{l}\text { French } \\
(\text { ATU })^{29}\end{array}$ & Italian $^{28}$ & Scottish $^{31}$ & Turkish $^{32}$ & Dutch $^{34}$ & Austrian $^{33}$ \\
\hline \multicolumn{9}{|l|}{$\begin{array}{l}\text { Baseline } \\
\text { characteristics }\end{array}$} \\
\hline$N$ & 49 & 179 & 282 & 20 & 42 & 25 & 90 & 155 \\
\hline$\%$ MDS & 69.4 & 63 & 78 & 85 & 45.2 & 48.0 & 52.2 & 0 \\
\hline$\%$ AML & 26.5 & 31 & 22 & 15 & 45.2 & 32.0 & 34.4 & 100 \\
\hline$\%$ CMML & 4.1 & 6 & 0 & 0 & 9.5 & 20.0 & 13.3 & 0 \\
\hline $\begin{array}{l}\text { Age (years), } \\
\text { median }\end{array}$ & 74.7 & 69 & 71 & 71 & 72 & 70 & 71 & 73 \\
\hline \multirow{2}{*}{\multicolumn{9}{|c|}{$\begin{array}{l}\text { Efficacy } \\
\text { Treatment } \\
\text { response }\end{array}$}} \\
\hline & & & & & & & & \\
\hline$N$ & $\begin{array}{l}38 \\
(29 \text { for } \mathrm{HI})\end{array}$ & $\begin{array}{l}179 \\
(177 \text { for } \mathrm{HI})\end{array}$ & 282 & 20 & 42 & 25 & 90 & 155 \\
\hline $\mathrm{CR}^{+}+\mathrm{PR}+\mathrm{HI}, \%$ & 42.1 & 78 & 32 & 50 & 31.0 & 64.0 & 48 & 45.2 \\
\hline $\mathrm{CR}^{+}, \%$ & 18.4 & 17 & 14 & NR & 9.5 & 12.0 & 26 & 12.9 \\
\hline PR, \% & 7.9 & 12 & 3 & NR & 0.0 & 12.0 & 1 & 20.6 \\
\hline $\mathrm{HI}, \%$ & 12.2 & 49 & 15 & NR & 21.5 & 40.0 & 21 & 9.0 \\
\hline SD, \% & 39.5 & 42 & 22 & 20 & NR & 44.0 & 19 & $2.6^{\dagger}$ \\
\hline $\begin{array}{l}\text { Follow-up, } \\
\text { median (months) }\end{array}$ & 9 & 21.1 & $26 / 41.3^{*}$ & NR & 6.0 & 13 & 8 & NR \\
\hline OS, median & $490 \mathrm{~d}(16.1 \mathrm{~m})$ & $24.5 \mathrm{~m}$ & $13.5^{*} \mathrm{~m}$ & $356 \mathrm{~d}(11.7 \mathrm{~m})$ & NR & NA & $13.0 \mathrm{~m}$ & $9.8 \mathrm{~m}$ \\
\hline
\end{tabular}

Note: ATU, authorization for temporary utilization; $N$, total number of patients; \%, percentage of patients within the category; MDS, myelodysplastic syndromes; AML, acute myeloid leukaemia; CMML, chronic myelomonocytic leukaemia; CR, complete response; PR, partial response; HI, haematological improvement; SD, stable disease; OS, overall survival; d, days; m, months; NR, not reported; NA, not assessed.

*Additional analysis of the study: Itzykson et al. (2012). ${ }^{30}$

†Marrow SD with HI.

\$CR or CR/CRi (CR with incomplete blood count recovery) or CR/CRi/mCR (marrow CR). 
study, respectively (Table 3 ). ${ }^{20,28,29,34}$ The longer median OS in the AZA-001 trial probably resulted from the more stringent eligibility criteria of clinical trials compared with real-life studies. In our survey, patients considered as responders (CR, PR, or $\mathrm{HI})$ and patients with SD seemed to have longer survival than nonresponders (19.2 and 17.1 months vs. 8.9 months). Similarly, in the Dutch study, the OS of responders was significantly longer than that of non-responders (16 months vs. 6 months; $P<0.001$ ), whereas in the Austrian study, any kind of response resulted in significantly longer OS compared to patients without such response, i.e. marrow response $(24.7$ months for CR vs. 15.2 months for marrow SD vs. 2.3 months for PD) or HI (18.9 months vs. 6.0 months). ${ }^{33,34}$ Furthermore, an additional analysis of the AZA-001 study evaluating the relationship between treatment response and OS revealed that OS of patients with treatment response or HI was significantly improved compared to non-responders; the OS was also significantly higher in patients treated with azacitidine than in those treated with conventional care. ${ }^{35}$

The main limitation of our survey is the small number of patients included and a relatively short follow-up period (1 year). Our findings describe the safety and efficacy of azacitidine treatment in this group of patients and must be interpreted cautiously. Our data are in line with those from previous real-life studies and support findings from trials that suggest that azacitidine prolongs the survival of MDS, AML, and CMML patients.

\section{Disclaimer Statements}

Contributors All authors contributed to the conception, design, acquisition, or generation of data or analyses, patient enrolment, interpretation of data for the publication, drafting the article, or revising it critically for important intellectual content and final approval or the version to be published.

Funding Celgene Corporation, Summit, NJ was the funding source and was involved in all stages of the study conduct and analysis. The Celgene Corporation also took responsibility for all costs associated with the development and publishing of the present manuscript.

Conflicts of interest YB, DS, SM, CG, GB, DD, IV, $\mathrm{CR}, \mathrm{KT}, \mathrm{FT}, \mathrm{LN}$, and PM received investigators fees in the context of this study. YB received grants and consulting fees from Celgene during the conduct of the study. DS received consulting fees from Celgene during the conduct of the study, and consulting fees and non-financial support from Celgene outside the submitted work. SM received consulting fees from Celgene during the conduct of the study. GB and PM received grants from Celgene during the conduct of the study. HP was an employee of Celgene at the time of manuscript praparation. $\mathrm{VV}$ is an employee of Celgene. HP and VV are stock holders of Celgene. $\mathrm{CG}, \mathrm{DD}, \mathrm{CR}, \mathrm{KT}$, and $\mathrm{LN}$ declare no conflict of interest.

Ethics approval The protocol and associated documents were reviewed and approved by the National Competent Authority and National/Local Ethics Committees in accordance with local regulations.

\section{Acknowledgements}

The authors thank study participants and their families and the following investigators: Dr Pierre Pascal, the principal investigator from Clinique Saint-Joseph (Arlon, Belgium), Dr Helene Petre (data manager) from Polyclinique Jolimont (Haine-SaintPaul, Belgium), Dr Tom Lodewyck (co-investigator), and Annelies Sneppe (data manager) from AZ SintJan hospital (Brugge-Oostende, Belgium). Finally, the authors thank Urszula Miecielica, PhD (XPE Pharma \& Science) for providing medical writing services and editorial support in preparing this manuscript.

\section{References}

1 Scott BL, Deeg HJ. Myelodysplastic syndromes. Annu Rev Med. 2010;61:345-58.

2 Tefferi A, Vardiman JW. Myelodysplastic syndromes. N Engl J Med. 2009;361(19):1872-85

3 Vardiman JW. The World Health Organization (WHO) classification of tumors of the hematopoietic and lymphoid tissues: an overview with emphasis on the myeloid neoplasms. Chem Biol Interact. 2010;184(1-2):16-20.

4 Vardiman JW, Thiele J, Arber DA, Brunning RD, Borowitz MJ, Porwit A, et al. The 2008 revision of the World Health Organization (WHO) classification of myeloid neoplasms and acute leukemia: rationale and important changes. Blood. 2009;114(5):937-51.

5 Cogle CR, Craig BM, Rollison DE, List AF. Incidence of the myelodysplastic syndromes using a novel claims-based algorithm: high number of uncaptured cases by cancer registries. Blood. 2011;117(26):7121-5.

6 Gattermann N, Kündgen A, Kellermann L, Zeffel M, Paessens $\mathrm{B}$, Germing $\mathrm{U}$. The impact of age on the diagnosis and therapy of myelodysplastic syndromes: results from a retrospective multicenter analysis in Germany. Eur J Haematol. 2013;91(6): 473-82.

7 Stauder R. The challenge of individualised risk assessment and therapy planning in elderly high-risk myelodysplastic syndromes (MDS) patients. Ann Hematol. 2012;91(9):1333-43.

8 Khan C, Pathe N, Fazal S, Lister J, Rossetti JM. Azacitidine in the management of patients with myelodysplastic syndromes. Ther Adv Hematol. 2012;3(6):355-73.

9 Disperati P, Ichim CV, Tkachuk D, Chun K, Schuh AC, Wells RA. Progression of myelodysplasia to acute lymphoblastic leukaemia: implications for disease biology. Leuk Res. 2006;30(2):233-9.

10 Fullmer A, Borthakur G, Kadia TM, Garcia-Manero G, Jabbour E. Prognostic factors associated with progression of myelodysplastic syndromes (MDS) to acute myeloid leukemia (AML) in patients (pts) treated with decitabine. J Clin Oncol. 2010;28:15s (suppl; abstr 6571).

11 Greenberg PL, Attar E, Bennett JM, Bloomfield CD, de Castro CM, Deeg HJ, et al. NCCN Clinical Practice Guidelines in Oncology: myelodysplastic syndromes. J Natl Comsupr Canc Netw. 2011;9(1):30-56.

12 Bennett JM, Catovsky D, Daniel MT, Flandrin G, Galton DA, Gralnick $\mathrm{H}$, et al. The chronic myeloid leukaemias: guidelines for distinguishing chronic granulocytic, atypical chronic mye- 
loid, and chronic myelomonocytic leukaemia. Proposals by the French-American-British Cooperative Leukaemia Group. Br J Haematol. 1994;87(4):746-54.

13 Estey E, Döhner H. Acute myeloid leukaemia. Lancet. 2006;368:1894-907.

14 Al-Ali HK, Brand R, van Biezen A, Finke J, Boogaerts M, Fauser AA, et al. A retrospective comparison of autologous and unrelated donor hematopoietic cell transplantation in myelodysplastic syndrome and secondary acute myeloid leukemia: a report on behalf of the Chronic Leukemia Working Party of the European Group for Blood and Marrow Transplantation (EBMT). Leukemia. 2007;21(9):1945-51.

15 Bejar R, Tiu RV, Sekeres MA, Komrokji RS. Myelodysplastic syndromes: recent advancements in risk stratification and unmet therapeutic challenges. Am Soc Clin Oncol Educ Book. 2013;2013:e256-70.

16 de Witte T, Hermans J, Vossen J, Bacigalupo A, Meloni G, Jacobsen N, et al. Haematopoietic stem cell transplantation for patients with myelo-dysplastic syndromes and secondary acute myeloid leukaemias: a report on behalf of the Chronic Leukaemia Working Party of the European Group for Blood and Marrow Transplantation (EBMT). $\mathrm{Br} \quad \mathrm{J}$ Haematol. 2000;110(3):620-30.

17 Kroger N, Zabelina T, Guardiola P, Runde V, Sierra J, van Biezen A, et al. Allogeneic stem cell transplantation of adult chronic myelomonocytic leukaemia. A report on behalf of the Chronic Leukaemia Working Party of the European Group for Blood and Marrow Transplantation (EBMT). Br J Haematol 2002;118(1):67-73.

18 Giralt SA, Horowitz M, Weisdorf D, Cutler C. Review of stemcell transplantation for myelodysplastic syndromes in older patients in the context of the Decision Memo for Allogeneic Hematopoietic Stem Cell Transplantation for Myelodysplastic Syndrome emanating from the Centers for Medicare and Medicaid Services. J Clin Oncol. 2011;29(5):566-72.

19 Mufti GJ, Potter V. Myelodysplastic syndromes: who and when in the course of disease to transplant. Hematology Am Soc Hematol Educ Program. 2012;2012:49-55.

20 Fenaux P, Mufti GJ, Hellstrom-Lindberg E, Santini V, Finelli C, Giagounidis A, et al. Efficacy of azacitidine compared with that of conventional care regimens in the treatment of higherrisk myelodysplastic syndromes: a randomised, open-label, phase III study. Lancet Oncol. 2009;10(3):223-32.

21 Meers S, Selleslag D, Potier H, Glasmacher A, Mineur P Voelter V. Azacitidine access program for Belgian patients with myelodysplastic syndromes, acute myeloid leukemia or chronic myelomonocytic leukemia. Curr Med Res Opin. 2014; DOI: 10.1185/03007995.2014.972499, Epub ahead of print.

22 Oken MM, Creech RH, Tormey DC, Horton J, Davis TE, McFadden ET, et al. Toxicity and response criteria of the Eastern Cooperative Oncology Group. Am J Clin Oncol. 1982;5(6):649-55.

23 Greenberg P, Cox C, LeBeau MM, Fenaux P, Morel P, Sanz G, et al. International scoring system for evaluating prognosis in myelodysplastic syndromes. Blood. 1997;89(6):2079-88.

24 US Department of Health and Human Services. Common terminology criteria for adverse events (CTCAE) v4.0 [cited 2013 Nov 12]. Available from: http://evs.nci.nih.gov/ftpl/ CTCAE/CTCAE_4.03_2010-06-14_QuickReference_5x7.pdf
25 Cheson BD, Bennett JM, Kantarjian H, Pinto A, Schiffer CA, Nimer SD, et al. Report of an international working group to standardize response criteria for myelodysplastic syndromes. Blood. 2000;96(12):3671-4.

26 Cheson BD, Bennett JM, Kopecky KJ, Buchner T, Willman $\mathrm{CL}$, Estey EH, et al. Revised recommendations of the International Working Group for Diagnosis, Standardization of Response Criteria, Treatment Outcomes, and Reporting Standards for Therapeutic Trials in Acute Myeloid Leukemia. J Clin Oncol. 2003;21(24):4642-9.

27 Silverman LR, Demakos EP, Peterson BL, Kornblith AB, Holland JC, Odchimar-Reissig R, et al. Randomized controlled trial of azacitidine in patients with the myelodysplastic syndrome: a study of the cancer and leukemia group B. J Clin Oncol. 2002;20(10):2429-40.

28 Greco R, Riva M, Ravano E, Molteni A, Morra E. Azacitidine in high-risk MDS patients. A 'real life' experience from a single center. In: Proceedings of the 12th Biennial International Symposium on Myelodysplastic Syndromes 2013; 2013 May 811; Berlin, Germany. Leukemia Research vol 37, Supplement 1, p S157, May 2013 (P-299) http://www.lrjournal.com/article/ S0145-2126(13)70346-1/abstract

29 Itzykson R, Thepot S, Quesnel B, Dreyfus F, Beyne-Rauzy O, Turlure $\mathrm{P}$, et al. Prognostic factors for response and overall survival in 282 patients with higher-risk myelodysplastic syndromes treated with azacitidine. Blood. 2011;117(2):403-11.

30 Itzykson R, Thepot S, Quesnel B, Dreyfus F, Recher C, Wattel $\mathrm{E}$, et al. Long-term outcome of higher-risk MDS patients treated with azacitidine: an update of the GFM compassionate program cohort. Blood. 2012;119(25):6172-3.

31 Lund K, Drummond M, Eynaud P, Fitzsimons K, Olaiya A, Copland M. Real word use of azacitidine in elderly patients with MDS/AML - the Scottish experience. In: Proceedings of the 18th Congress of the European Hematology Association (EHA); 2013 Jun 13-16; Stockholm, Sweden. The Hague: EHA; 2013. Abstract Book. Haematologica June 2013; 98:1-768 http://www.haematologica.org/content/98/supplement_1/1.fulltext.pdf + html

32 Ozbalak M, Cetiner M, Bekoz H, Atesoglu EB, Ar C, Salihoglu A, et al. Azacitidine has limited activity in 'real life' patients with MDS and AML: a single centre experience. Hematol Oncol. 2012;30(2):76-81.

33 Pleyer L, Stauder R, Burgstaller S, Schreder M, Tinchon C, Pfeilstocker M, et al. Azacitidine in patients with WHO-defined AML - results of 155 patients from the Austrian Azacitidine Registry of the AGMT-Study Group. J Hematol Oncol. 2013;6(1):32.

34 van der Helm LH, Alhan C, Wijermans PW, van Marwijk Kooy M, Schaafsma R, Biemond BJ, et al. Platelet doubling after the first azacitidine cycle is a promising predictor for response in myelodysplastic syndromes (MDS), chronic myelomonocytic leukaemia (CMML) and acute myeloid leukaemia (AML) patients in the Dutch azacitidine compassionate named patient programme. Br J Haematol. 2011;155(5):599-606.

35 Gore SD, Fenaux P, Santini V, Bennett JM, Silverman LR, Seymour JF, et al. A multivariate analysis of the relationship between response and survival among patients with higher-risk myelodysplastic syndromes treated within azacitidine or conventional care regimens in the randomized AZA-001 trial. Haematologica. 2013;98(7):1067-72. 